\title{
Speed control of subways and trams
}

\author{
I. Strainescu, E. Tudor, V. Serbu, F. Bozas \& S. Badea \\ ICPE SAERP S.A., Bucharest, Romania
}

\begin{abstract}
In this paper we present the speed control system developed at ICPE SAERP SA Bucharest for traction equipment consisting of more than one drive, such as the two-motored tramcar, the double-unit metro or the tractor coach for railway use. The system contains hierarchical processor controllers for speed and torque. The main connections between processor units are serial connections, and the communication protocol has special safety tasks. The speed regulators are based on velocity measurements of the axles and are located at main controllers in order to operate at low frequency. Each of the motor units is driven with individual power converters. Inside the power converter is located a slave processor which performs the current regulation based on the local transducers. The diagnosis tasks are both global with master controller and local by every slave controller, so the central diagnosis task can operate the data with high resolution. The system allows the addition of several motored units and can be driven from a selectable cabin. Further converter and motor evolution will easily fit inside the system.
\end{abstract}

Keywords: speed control, tram, metro, converter, diagnosis.

\section{Introduction}

Due to operating characteristics and requirements, big urban transport vehicles fully meet basic transport requirements, such as short stations, maximum speed restriction of approximately $70-80 \mathrm{~km} / \mathrm{h}$, large traffic of passengers and the possibility of organizing a good complex system of transport. Thus, we consider that, at the present moment, electrical transport based on trams and metro trains in the urban area and by trains in the suburban area, represents the best solution.

In Bucharest as well as in the other towns in our country, the transport systems have been recently reconsidered by considering the metro and the trams as basic means of urban transport. That is the reason why, at present, all efforts 
are meant to contribute to the transport network and wagon park extension and to the modernization and implementation, on these vehicles, of new technologies for the control systems.

ICPE SAERP Bucharest, in cooperation with "ASTRA VAGOANE" Arad (Wagon Factory) - the metro general designer - and "METROREX" (metro operating enterprise Bucharest) have agreed on the refurbishment of the present metro cars, by using choppers and DC motors.

During the same period ICPE SAERP, in cooperation with "Electrometal" Timisoara (tram wagon manufacturer), have agreed on tram refurbishment using choppers.

These drives provide an economic start without start resistances and energy recuperation braking, easily obtaining the control of the train formed by several rail cars. In the circumstances, for ICPE SAERP, the problem was to perform a multiple control system for subway and tram wagons, which uses most of the subassemblies and microprocessors in common.

Concerning the subway frames design, we have undertaken the following subject conditions:

$\checkmark \quad$ To provide control over the train, a medium voltage line (110 Vdc for subway and $60 \mathrm{Vdc}$ for the trams);

$\checkmark$ Each wagon should have independent driving so that in the case of damage, the faulty wagon could be electrically isolated;

$\checkmark \quad$ To provide sure and simple slipping protection when accelerating and wheel blocking protection during braking;

$\checkmark \quad$ The basic braking should be electrical with energy recuperation and it should be completed by rheostat electrical braking;

$\checkmark$ The possibility of proving the automatic train stop at fixed points and the subsequent introduction of the autopilot;

$\checkmark \quad$ The possibility of the control assembly diagnostic.

As far as the tram is concerned there are same specific conditions, namely:

$\checkmark \quad$ The possibility of bimotor bogie equipped wagon control;

$\checkmark \quad$ The possibility of providing, in the case of the single rail car traffic, an electrical isolation of the faulty bogie and the driving by means of a valid motor bogie.

\section{Operating principle of train control}

In order to provide a centralized control in the case where the train imposed speed transmission represents the basic value, the hierarchical control system was conceived to operate in a three level architecture. For the train control, at the higher hierarchical level, there is active pilot block (PB) in the command post of the machinist.

Fig. 1 shows the main active PB connections to the other train equipment from which the PB receives and permanently analyses the information and then transmits to the train bus the basic command for the train control, including first the time-imposed speed. 


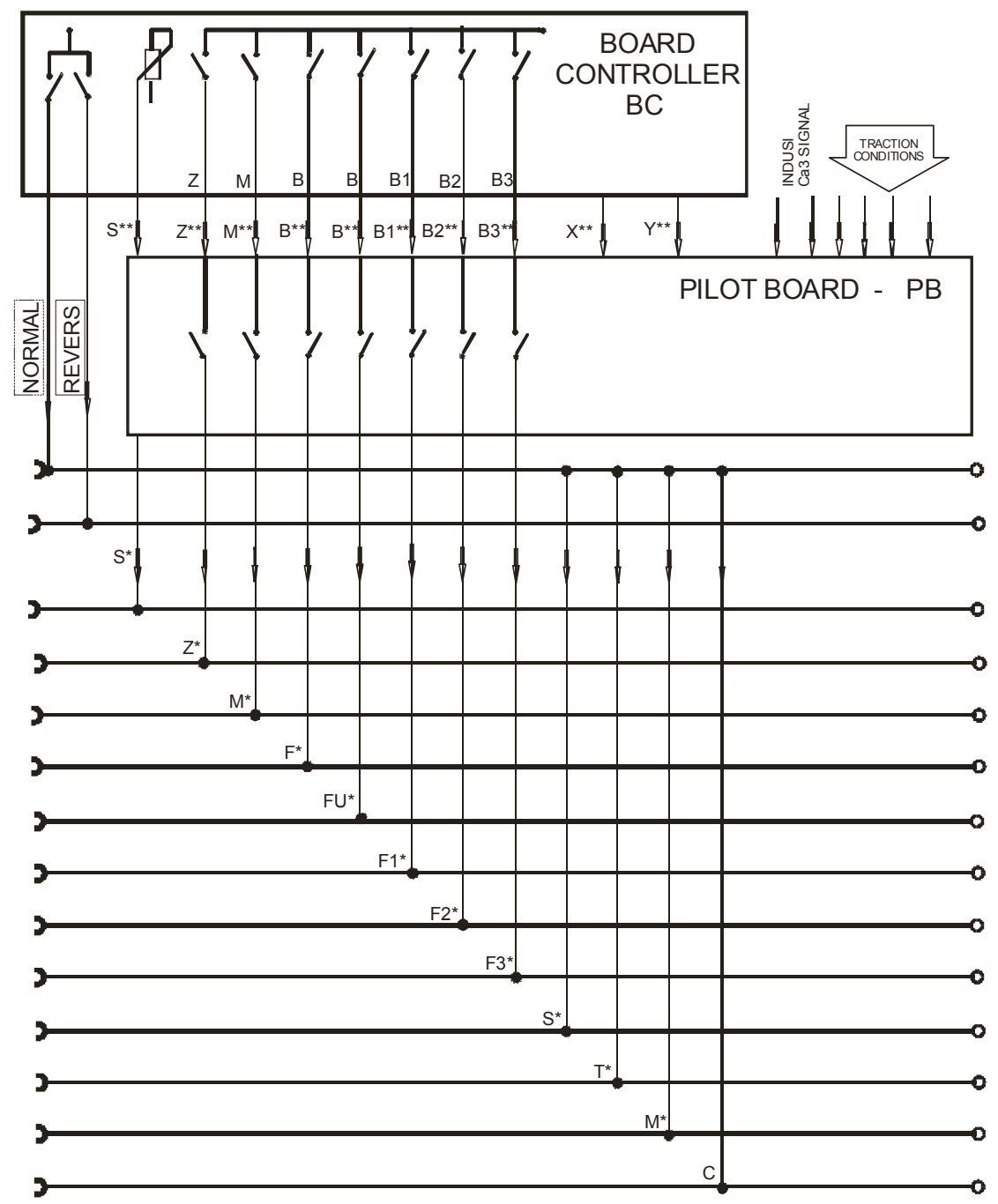

Figure 1: Logical interconnections between the active $\mathrm{BC}$, the active $\mathrm{PB}$, the traffic safety equipment and the exit signals equipments.

The machinist intervenes in the train control through the active board controller (BC) in the command post of the machinist. The $\mathrm{BC}$ has two mechanically interlocking handles.

The first handle is used to control the train's forward running and the possible running speed limits (the values $\mathrm{X}$ and $\mathrm{Y}$ for the subway limit the maximum train speed at $5 \mathrm{~km} / \mathrm{h}$ and $15 \mathrm{k} / \mathrm{h}$, respectively, depending on the special running regimes: frame coupling, pantograph running, signal limited over speed, etc.). 
The second handle controls the normal regimes, fast running, braking and stopping. An attached position transducer provides the output signal $\mathrm{S}^{* *}$ proportional to the handle axle displacement angle. In the running regime, $\mathrm{S}^{* *}$ represents the maximum speed required by the machinist and, in the braking regime, the required deceleration.

The PB transmits the logical running commands to the bus, if the machinist required it, by controller command and if the running conditions are met for the whole train (closed doors, correct air-pressure, unbraked train, etc.).

The braking command can be given to the machinist by the automatic controller when the traffic safety equipment interferes.

The main braking system is the electrical braking system with energy regeneration, completed with electrical resistive brake. In the case that one of the wagon's electrical brakes is not efficient, the pneumatic brake is automatically engaged, proportionally to the braking handle displacement angle (B1, B2, B3) and in the case of the necessity of emergency braking (EB); the pneumatic braking put-in decision is taken by the control block of each wagon.

Besides the logical signals R, Z, B, etc., the PB transmits the basic signal S* in terms of digital dates or variable frequency pulses. In the running regime, $\mathrm{S}^{*}$ follows $\mathrm{S}^{* *}$ with the acceleration limiting corrections of the first and second order, as shown in Fig.2(a).
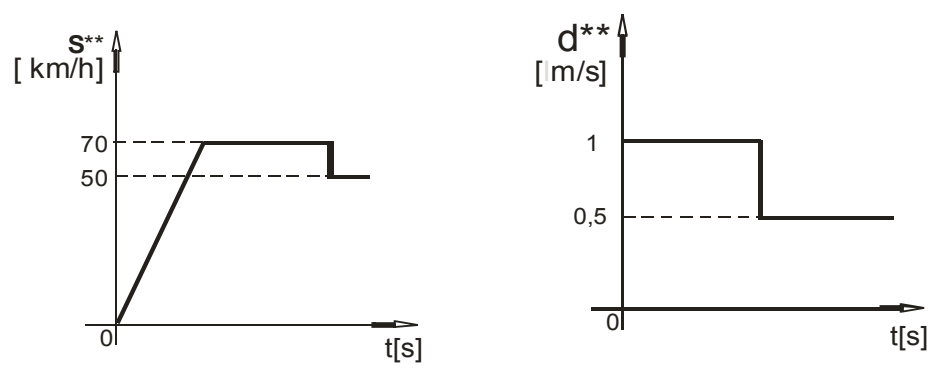

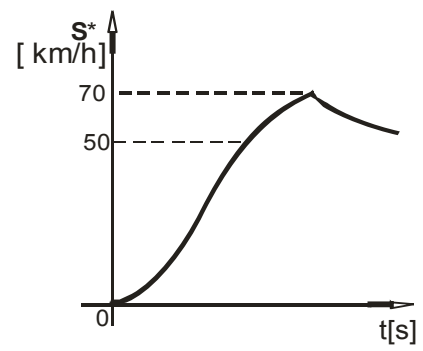

(a)

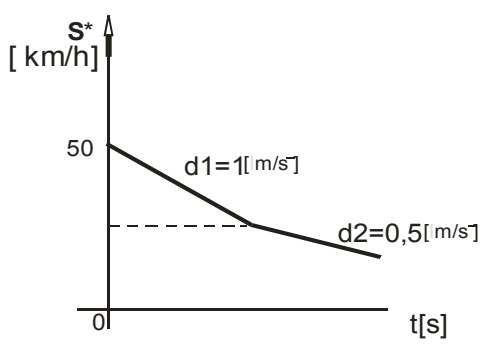

(b)

Figure 2: Characteristics: (a) for traction; (b) for brake. 
In the braking regime $\mathrm{S}^{*}$ starts from the train speed before the braking and decreases with deceleration, proportional to the signal $\mathrm{S}^{* *}$ value, as shown in Fig.2(b). In the case whereby the EB or the B3 braking were commanded from the traffic safety devices, the $\mathrm{PB}$ will release an output signal $\mathrm{S} *$ with a deceleration function of $\mathrm{OB}$ or B3 braking.

As for the subway chopper cars, the $\mathrm{T}$ and $\mathrm{M}$ signals are transmitted in terms of digital or pulses, these signals representing the operating tact of the train chopper image pulses (Fig.3).

These pulses must have a very constant frequency. For each wagon the $\mathrm{T}$ pulses are selected as a function of the wagon position relative to the train. The suitable pulses are selected so that the two choppers of each wagon should have the $\mathrm{T} / 2$ pulses, from $\mathrm{T}$ total period. Thus, for the feeder line, the train receives the current with minimum undulations.
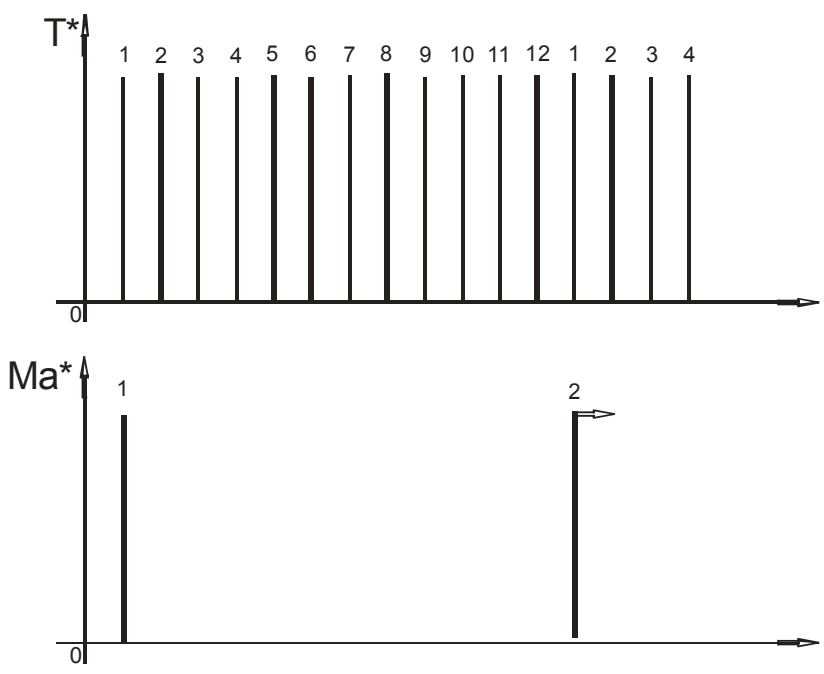

Figure 3: $\quad$ The $\mathrm{T}^{*}$ and $\mathrm{Ma}$ * control pulses, as a function of time, transmitted to the line.

\section{Operating principle of the wagon control system}

According to the speed imposed multiple control concept conceived within ICPE SAERP, each wagon has a micro controlling wagon block (CWB), which operates independently from the other block. The CWB receives input signals from the train bus and from its own wagon (the motor axle speed, the electrical braking existence signal, etc.) (Fig.4).

This block performs the correction speeds $\mathrm{S} 1^{*}$ and $\mathrm{S} 2 *$ and transmits them to the bogie1 and bogie 2 control blocks, in comparison with the value $\mathrm{S}^{*}$ received from the train bus as a function of the diameter of the driving wheel. For the subway wagon, it is possible for this correction to be automatically performed during fast running when comparing the speeds S1 and S2 from each bogie of 
the subway's own wagon with the speed measured at the standard bogie which is transmitted in the train bus, the correction being stored until a new fast running.

The CWB also provides the force pattern formation by means of the corresponding contactor and electro valve command, in the case of the pneumatic braking command.

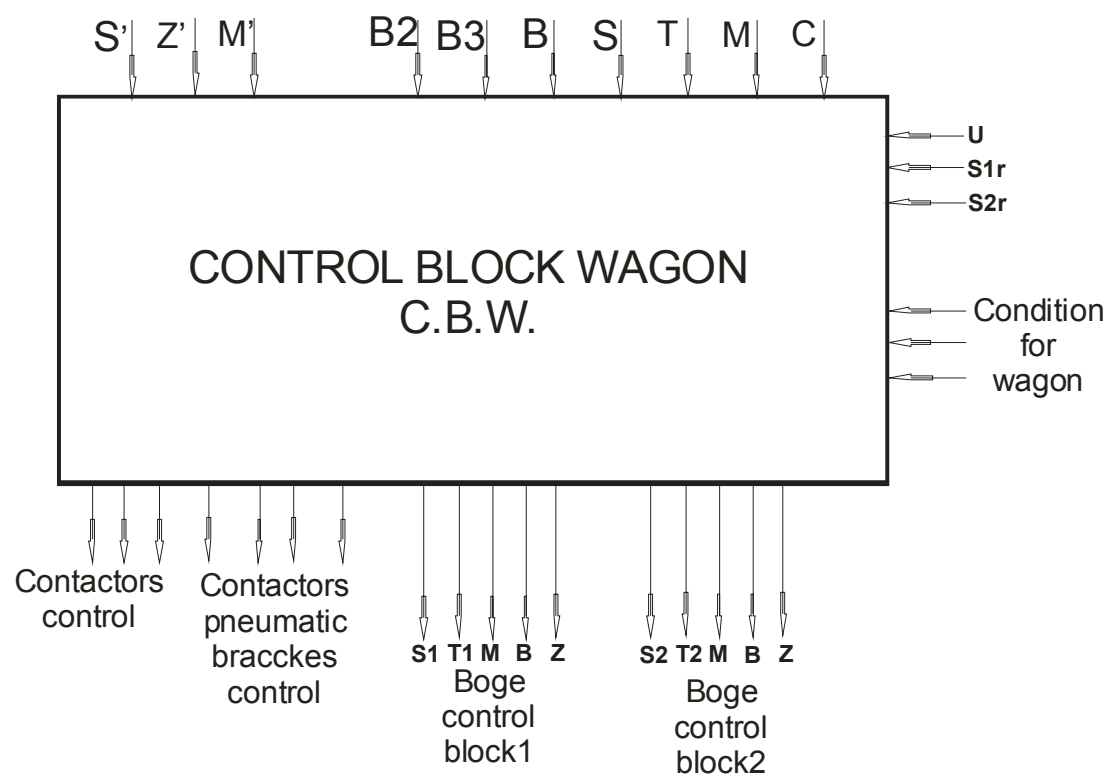

Figure 4: The block pattern of information processing by the control wagon block.

To the control bogie block (CBB) comes on the one hand the control signals from the higher hierarchical CB block (R,B,Z, T signals) and on the other hand the speed for the bogie $\mathrm{Sr} 1$ and real current information from the traction motor and $\mathrm{S} 1 *$ for the first bogie, from the CWB.

During the traction regime (Fig.5) the speed regulator, Reg S1, is activated and it receives as a positive value the value $\mathrm{S} 1^{*}$ and as feedback the bogie real speed Sr1. During the braking regime, the speed regulator Reg S2 is activated and it receives as feedback the imposed speed $\mathrm{S} 1^{*}$ (as a reaction), and as a positive value the real speed $\mathrm{Sr} 1$ (which is higher as an absolute value than $\mathrm{S} 1 *$ ).

The output of Reg S1 or Reg S2 is a positive input (imposed value) for current regulator Reg I. and the feedback is the motor current. The current regulator output value goes to DCT for IGBT transistor control.

\section{Train control by means of the imposed speed}

In order to provide optimum running for a train formed by motor wagons it is necessary to perform a high first order acceleration without exceeding the maximum admitted value for the second order acceleration; and for the braking 
to provide the PB controlled deceleration, irrespective of the wagon load, the routing aspect and without providing by means of it's own motors, a speed near to speed $\mathrm{S}^{*}$.
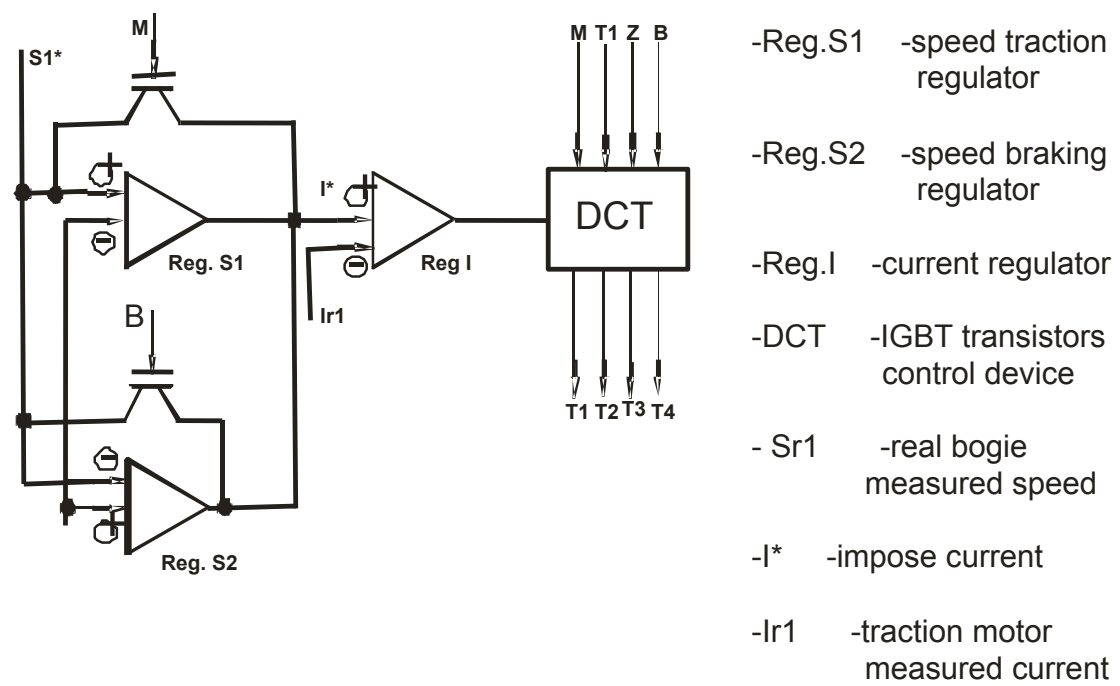

Figure 5: The principle pattern of the CBB.

On the other hand, the train imposed speed $\mathrm{S}^{*}$ must be permanently calculated by the PB so that the maximum motor admitted current should not be exceeded in any situation, and the current regulator should operate as rarely as possible under normal operating condition.

The speed together with other auxiliary commands transmitted to the signal line by the PB, are finally received by the CBB (Fig.6). As a matter of fact each CBB operates independently under normal operating conditions, it's output values depending on the information processing performed by it's own current and speed regulators.

The control by $\mathrm{S}^{*}$ may be used within the autopilot command system, in this case the $\mathrm{S}^{*}$ function being recorded as a function of time, necessary for each inter station or transmitted to the railway $\mathrm{S}^{*}$ from the train automatic monitoring center, the exact stoppage being provided by the stopping system at a fixed point.

The imposed speed control $\mathrm{S}^{*}$ also provides the start slipping protection and the wheel blocking during braking, respectively. If there are bogie wheels slipping at start, the regulator that compares $\mathrm{S}^{*}$ to $\mathrm{Sr}$ from the axle speed transducer controls the chopper so that that the chopper output voltage (Fig. 6) should properly decrease. In this way, the slipping is limited and can be removed if the other bogies can compensate for the lack of the bogie slipping traction forces.

During braking, an axle blocking, which breaks the adherence, is removed by putting into operation the current regulator, which will impose a decreased braking current. 


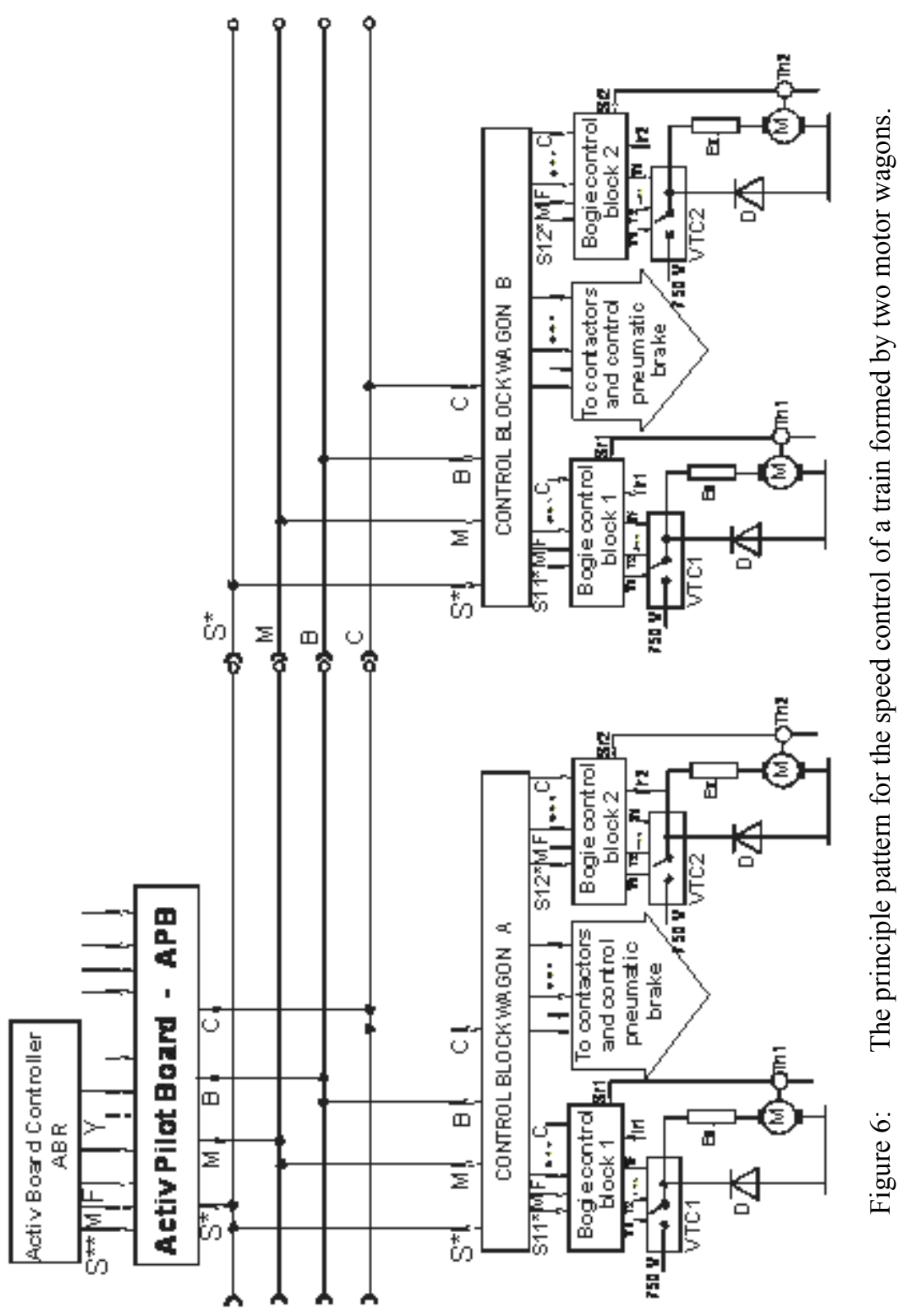




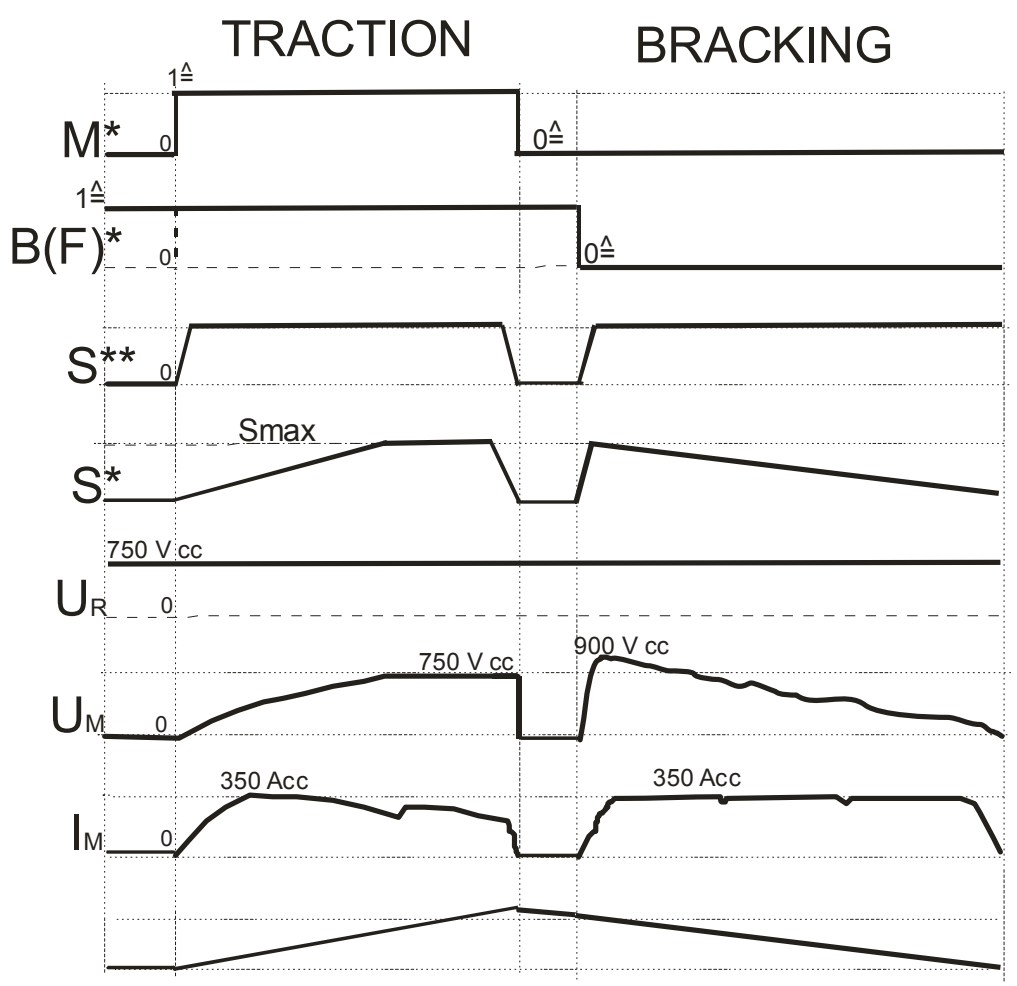

Figure 7: The main dynamic values oscillograms.

\section{Performances obtained with the Romanian imposed speed control vehicles}

The "Astra Vagoane" Arad (RO) Company has realized two chopper subway trains. The chopper driving equipment and the control blocks (PB, WCB, CBB) have been designed and produced by ICPE SAERP Bucharest and final prototype homologation tests have been carried out. The tests conclude a uniform loading of all traction motors and good dynamic performances without shocks between wagons. During the test carried out under water and soap lubricated rail conditions, the correct operation of the protection system, necessary in wheels slipping and blocking was confirmed.

Additionally, there was a certificated tram prototype TIMIS-1 equipped with chopper and DC motors. This tram was equipped with two motored bogies, each of them being driven by two DC motors. Fig.7 shows the scope record containing the main parameters obtained in the traction and braking fields for the $\mathrm{S}^{*}$ speed controlled tram. From the scope diagrams it results that the train's real speed follows imposed speed $\mathrm{S}^{*}$ both in traction and braking regimes, without exceeding the traction motors' admitted currents. 